\title{
Could good eczema care prevent development of other atopic conditions?
}

The 'atopic march' describes the progression from eczema to other atopic conditions, mainly asthma and allergic rhinitis, and there has been a sharp rise in prevalence of all these conditions over the past few decades. Recent research suggests that changes in the skin barrier in early life are central to the development of atopy and there is gathering evidence to suggest that control or prevention of eczema in infancy may prevent future development of other atopic conditions.

Genetic research has shed light on the susceptibility of some individuals to develop atopic conditions through defects in genes involved in skin barrier function. Several studies have demonstrated an increased risk of developing eczema in individuals with defects in the filaggrin gene, which is present in up to $10 \%$ of western European populations. ${ }^{1}$ Filaggrin is one of a number of structural proteins of the epidermis which contribute to maintaining an effective skin barrier. Mutations in the filaggrin gene result in loss of skin barrier function and the presence of atopic diseases, particularly eczema and asthma. Filaggrin gene defects are associated with an increased risk of developing asthma in individuals with eczema, but not those without eczema and, interestingly, filaggrin is not expressed in bronchial mucosa. Therefore, in individuals with filaggrin mutations, it seems that the breakdown in skin barrier plays a role in the development of asthma. ${ }^{2}$

It is hypothesised that lack of skin barrier function allows exposure to allergens through the skin and that the atopic march arises due to subsequent migration of sensitised $\mathrm{T}$ cells to the airways and nose. Mice models have shown that filaggrin mutations lead to increased uptake of allergens through the skin, contributing to increased immunoglobulin-E sensitisation. ${ }^{2}$ It is thought that eczema is aggravated by contact with soaps or detergents which can compromise the barrier function of the skin. Protection of the skin barrier by excellent eczema care in early stages of the disease may decrease the risk of developing other atopic conditions. ${ }^{3}$ Large prospective trials are needed to test this and it may mean that we need to improve eczema care radically in primary care where early disease is managed.

\section{IMPACT OF THE CONDITION}

Eczema causes significant distress to the child and family due to sleep disturbance and behavioural problems related to itch. ${ }^{4}$ Research from secondary care suggests that in terms of impact on quality of life in children, generalised eczema is second only to cerebral palsy, with greater impact reported than for asthma or diabetes. ${ }^{5}$ One study carried out among parents of infants with eczema in primary and secondary care reported that parents often felt the condition was not taken sufficiently seriously by practitioners. ${ }^{6}$ Parents felt that the advice they had received was inadequate and inconsistent and they wished for more reliable information about treatments, triggers, and nonpharmacological treatments, especially dietary advice.

Another study found that parents gave a number of reasons for not using topical treatments, such as poor understanding of treatments, child refusal, or therapy being too time-consuming. ${ }^{7}$ Others have found that the majority of carers do not feel confident that they can adequately manage their child's eczema. ${ }^{8}$

\section{TREATMENT APPROACHES}

Skin complaints are the second most common reason for GP consultation, after infectious illness, in children under the age of 5 years. ${ }^{9}$ Primary care is therefore ideally placed to promote improvements in eczema care, and the National Institute for Health and Clinical Excellence (NICE) guideline on atopic eczema in children ${ }^{10}$ provides a template for this. There is an emphasis on a stepped approach, paired with ongoing education of parents and carers, analogous to asthma care.

Emollients represent the first step of the care plan, and should be used even when the eczema is clear to reduce the frequency of flare-ups and protect the skin barrier. Carers need specific information on how to step up treatment during a flare-up, initially with increased frequency of emollient application and commencing mild topical corticosteroids, applied once or twice daily.

Mild topical corticosteroids should be used for mild eczema, moderate corticosteroids for moderate eczema. Mild potency only should be used for face and neck, or moderate for a severe flare-up for short-term use (3-5 days). Very potent topical corticosteroids should only be used in children in secondary care, and potent preparations only used in infants under 1 year of age in secondary care. In all children, potent topical corticosteroids should be used for short periods only (5-7 days). Those requiring longer treatments or frequent usage should receive dermatological advice.

The NICE guideline also provides a checklist of educational points to be covered in a regular review including: (1) identification and management of trigger factors, such as soaps (including shampoo), infections, or allergens; (2) advice on when and how to step treatment up and down; and (3) ensuring that treatment is still optimal.

The choice of emollient may change over time and should be prescribed and used in sufficient quantities (250-500 g weekly, with an offer of separate supplies to keep at school or nursery). Some emollients and bath oils may irritate skin, most commonly aqueous cream, which can be used as a soap substitute but not as a leave-on emollient.

Advice should be both verbal and written. Fortunately, good free sources of information are available, for instance Clinical 
Knowledge Summaries (www.cks.nhs.uk), the National Eczema Society (www.eczema.org), and the Nottingham Support Group for Carers of Children with Eczema (www.nottinghameczema.org.uk; which includes audio/podcast versions of leaflets).

\section{DIETARY AND OTHER CONSIDERATIONS}

GPs may find some aspects of the guideline controversial, such as the recommendation to consider food allergy in infants with moderate or severe atopic eczema that has not been controlled by optimum management, particularly if associated with gut dysmotility (colic, vomiting, altered bowel habit) or failure to thrive. The most common dietary allergens in infants are eggs, cow's milk, nuts, or soya, and suspected allergy is an indication for referral for allergy testing. Consumer testing including internet allergy tests should be discouraged, as their results are frequently misleading. The guideline suggests a 6-8-week trial of an extensively hydrolysed protein formula or amino acid formula in place of cow's milk formula for bottle-fed infants aged under 6 months with moderate or severe eczema who are not responding to adequate routine therapy or with gastrointestinal symptoms. Those staying on a cow's milk-free diet beyond 8 weeks should be referred for specialist dietary advice.

The NICE Guideline Development Group for atopic eczema in children reviewed the evidence on pets and house dust mites and did not strongly recommend avoidance on the basis of current knowledge. ${ }^{10}$ There is recent evidence suggesting that the presence of a dog in the house from birth may reduce the risk of development of eczema, but that cats may increase the risk. Removal of pets may have negative psychological consequences for older children. Evidence of benefit from house dust mite elimination strategies is poor, and such strategies were deemed impractical in many cases, although outcomes from further large trials are awaited. Exposure to maternal smoking in utero is associated with decreased lung function in childhood, ${ }^{11}$ suggesting that smoking interventions should reduce risk of children developing asthma in susceptible families.

\section{CHALLENGE TO PRIMARY CARE}

It is hoped that new genetic discoveries will lead to new treatments. However, in the meantime we have a renewed imperative to provide high quality treatment for childhood eczema. The challenge for primary care is to encourage carers to prioritise regular emollient use, while also reassuring them about the safety of topical corticosteroids if used correctly. The calls for eczema to be reviewed at least annually should be reassuring for GPs, as this does not represent new work, but suggests a structured approach to a medication review. The signposting of written materials and self-help groups (see above) may save time in the long term as improved self-care can improve outcomes and reduce healthcare use. ${ }^{12}$

Improved eczema care seems like an 'easy win' for primary care, representing possible prevention of a condition that costs distress to child and family and considerable time and funds to the NHS. Even if the hypothesis regarding the prevention of the atopic march remains unproven, better eczema care will improve quality of life for many children and their families.

\section{Miriam Santer,}

Clinical Research Fellow, Primary Medical Care, University of Southampton, Southampton.

\section{Sue Lewis-Jones,}

Consultant Dermatologist and Paediatric Dermatologist, Department of Dermatology, Ninewells Hospital \& Medical School, Dundee.

\section{Provenance}

Commissioned, not externally peer reviewed.

\section{REFERENCES}

1. O'Regan GM, Sandilands A, McLean WH, Irvine AD Filaggrin in atopic dermatitis. J Allergy Clin Immuno 2009; 124(3 Suppl 2): R2-R6.

2. Fallon PG, Sasaki T, Sandilands A, et al. A homozygous frameshift mutation in the mouse Flg gene facilitates enhanced percutaneous allergen priming. Nature Genetics 2009; 41: 602-608.

3. Van den Oord RA, Sheikh A. Filaggrin gene defects and risk of developing allergic sensitisation and allergic disorders: systematic review and meta-analysis. $B M J$ 2009; 339: b2433.

4. Chamlin SL, Frieden IJ, Williams ML, Chren M. Effects of atopic dermatitis on young American children and their families. Pediatrics 2004; 114(3): 607-611.

5. Beattie PE, Lewis-Jones MS. A Comparative study of impairment of Quality of Life (QoL) in children with skin disease and children with other chronic childhood diseases. Br J Dermatol 2006; 155: 145-151.

6. Gore C, Johnson RJ, Caress AL, et al. The information needs and preferred roles in treatment decision-making of parents caring for infants with atopic dermatitis: a qualitative study. Allergy 2005; 60(7): 938-943.

7. Beattie PE, Lewis-Jones MS. Parental knowledge of topical therapies in the treatment of childhood atopic dermatitis. Clin Exp Dermatol 2003; 28: 549-553.

8. Zuberbier T, Orlow SJ, Paller AS, et al. Patient perspectives on the management of atopic dermatitis. J Allergy Clin Immunol 2006; 118(1): 226-232.

9. McCormick A, Fleming D, Charlton J. Morbidity statistics from general practice. Fourth national study 1991-1992. London: HMSO, 1995.

10. National Institute for Health and Clinical Excellence. Atopic eczema in children. Management of atopic eczema in children from birth up to the age of 12 years. NICE clinical guideline 57. London: NICE, 2007.

11. Gilliland FD, Berhane K, McConnell R, et al. Maternal smoking during pregnancy, environmental tobacco smoke exposure and childhood lung function. Thorax 2000; 55(4): 271-276.

12. Greaves CJ, Campbell JL. Supporting self-care in general practice. Br J Gen Pract 2007; 57(543): 814-821

DOI: 10.3399/bjgp11X567018

ADDRESS FOR CORRESPONDENCE

Miriam Santer

University of Southampton, Primary

Medical Care, Aldermoor Health Centre,

Aldermoor Close, Southampton,

SO16 5ST.

E-mail:m.santer@soton.ac.uk 